\title{
Erratum to: Colloquium: Mechanical formalisms for tissue dynamics
}

Sham Tlili ${ }^{1}$, Cyprien Gay ${ }^{1,6, a}$, François Graner ${ }^{1,6, b}$, Philippe Marcq ${ }^{2}$, François Molino ${ }^{3,4,6}$, and Pierre Saramito ${ }^{5,6}$

${ }^{1}$ Laboratoire Matière et Systèmes Complexes, Université Denis Diderot - Paris 7, CNRS UMR 7057, 10 rue Alice Domon et Léonie Duquet, F-75205 Paris Cedex 13, France

2 Laboratoire Physico-Chimie Curie, Institut Curie, Université Marie et Pierre Curie - Paris 6, CNRS UMR 168, 26 rue d'Ulm, F-75248 Paris Cedex 05, France

3 Laboratoire Charles Coulomb, Univ. Montpellier II, CNRS UMR 5221, Place Eugène Bataillon, CC070, F-34095 Montpellier Cedex 5, France

4 Institut de Génomique Fonctionnelle, Univ. Montpellier I, Univ. Montpellier II, 141 rue de la Cardonille, CNRS UMR 5203, INSERM UMR_S 661, F-34094 Montpellier Cedex 05, France

${ }^{5}$ Laboratoire Jean Kuntzmann, Université Joseph Fourier - Grenoble I, CNRS UMR 5524, BP 53, F-38041 Grenoble Cedex, France

6 Academy of Bradylogists - Paris Cedex 13, France

Original article: Eur. Phys. J. E (2015) 38: 33, DOI: 10.1140/epje/i2015-15033-4

Received 1 October 2015

Published online: 30 October 2015 - C EDP Sciences / Società Italiana di Fisica / Springer-Verlag 2015

In appendix D.2.2, entitled "Constitutive equations", we have found a technical error concerning eqs. (D.37), (D.39): a rotation term was forgotten and the validity of these equations was erroneously not mentioned to be restricted to commuting tensors. This error has no consequence on the general validity of the appendix, although it slightly alters the resolution scheme. As a consequence, appendix D.2.2 should be slightly amended as follows:

\section{Modified appendix D.2.2}

In the small deformation expressions (4) or (8) of the dissipation function, the notation $\dot{\varepsilon}$ or $\dot{\varepsilon}_{1}$ in fact designate the symmetrised velocity gradient $D$ and its effective counterpart $D_{1}$ (see appendix D.1.4). Although this confusion has no consequence when the deformations $\varepsilon$ or $\varepsilon_{1}$ are small, see eqs. (D.23), (D.24), at large deformations it is necessary to express the dissipation function with respect to the correct kinematic variables:

$$
\begin{gathered}
\mathcal{D}=\mathcal{D}\left(D, D_{1}, \ldots, D_{m}\right), \\
\mathcal{D}=\mathcal{D}\left(\operatorname{tr} D, \operatorname{tr} D_{1}, \ldots, \operatorname{dev} D, \operatorname{dev} D_{1}, \ldots\right),
\end{gathered}
$$

while the static variables $\varepsilon, \varepsilon_{1}$ are still correct variables for the energy function.

The differentiation rule of the energy and dissipation function given by eqs. (B.10)-(B.13) is now rewritten using $D$ and $D_{1}$ :

$$
\begin{aligned}
\operatorname{dev} \sigma & =\frac{\partial \mathcal{D}}{\partial \operatorname{dev} D}+\frac{\partial \mathcal{E}}{\partial \widehat{\operatorname{dev} \varepsilon}}, \\
\operatorname{tr} \sigma & =\frac{\partial \mathcal{D}}{\partial \operatorname{tr} D}+\frac{\partial \mathcal{E}}{\partial \widehat{\operatorname{tr} \varepsilon}}, \\
0 & =\frac{\partial \mathcal{D}}{\partial \operatorname{dev} D_{1}}+\frac{\partial \mathcal{E}}{\partial \widehat{\operatorname{dev}} \varepsilon_{1}}, \\
0 & =\frac{\partial \mathcal{D}}{\partial \operatorname{tr} D_{1}}+\frac{\partial \mathcal{E}}{\partial \widehat{\operatorname{tr}} \varepsilon_{1}}
\end{aligned}
$$

\footnotetext{
${ }^{a}$ e-mail: cyprien.gay@univ-paris-diderot.fr

b e-mail: francois.graner@univ-paris-diderot.fr
} 
In eqs. (8b), (D.40)-(D.43), $\widehat{\operatorname{tr}} \varepsilon, \widehat{\operatorname{dev}} \varepsilon, \widehat{\operatorname{tr}} \varepsilon_{1}$ and $\widehat{\operatorname{dev}} \varepsilon_{1}$ are given by eqs. (D.29), (D.30). These equations are solved together with eq. (14) and yield directly the velocity field $\boldsymbol{v}$ (and its symmetrized gradient $D$ ), the effective symmetrized velocity gradient $D_{1}$ and the stress $\sigma$. The evolution of the deformations $\varepsilon$ and $\varepsilon_{1}$ and of the mass density $\rho$ is then obtained from eqs. (D.23), (D.24), (22).

Note that, as can be shown using eqs. (D.23), (D.24), (D.29)

$$
\begin{gathered}
\left(\partial_{t}+\boldsymbol{v} \cdot \nabla\right)[\widehat{\operatorname{tr}} \varepsilon]=\operatorname{tr} D \\
\left(\partial_{t}+\boldsymbol{v} \cdot \nabla\right)\left[\widehat{\operatorname{tr}} \varepsilon_{1}\right]=\operatorname{tr} D_{1} .
\end{gathered}
$$

In highly symmetric geometries such that the symmetrized velocity gradients $D$ and $D_{1}$ and the deformations $\varepsilon$ and $\varepsilon_{1}$ remain aligned, one can also show, using eqs. (D.23), (D.24), (D.30), that

$$
\begin{gathered}
\left(\partial_{t}+\boldsymbol{v} \cdot \nabla\right)[\widehat{\operatorname{dev}} \varepsilon]=\operatorname{dev} D+\Omega \widehat{\operatorname{dev} \varepsilon}-\widehat{\operatorname{dev} \varepsilon} \Omega, \\
\left(\partial_{t}+\boldsymbol{v} \cdot \nabla\right)\left[\widehat{\operatorname{dev}} \varepsilon_{1}\right]=\operatorname{dev} D_{1}+\Omega \widehat{\operatorname{dev}} \varepsilon_{1}-\widehat{\operatorname{dev}} \varepsilon_{1} \Omega .
\end{gathered}
$$

Whenever $D$ and $\varepsilon$ (or $D_{1}$ and $\varepsilon_{1}$ ) do not commute, eqs. (D.37), (D.39) cease to be valid.

In appendix D.2.3 entitled "Simple example", as a result of the restricted validity of eqs. (D.37), (D.39), the two sentences immediately after eqs. (D.46)-(D.49) should be amended as follows:

\section{Modified sentence in appendix D.2.3}

Equations (D.23)-(D.24), (D.29)-(D.30), and (D.46)-(D.49) are sufficient to describe the material evolution using a closed set of equations.

In appendix D.2.4 entitled "Complex example", consistently with the remark in appendix D.2.2, the small deformation notation $\dot{\varepsilon}_{\mathrm{ck}}$ must be replaced with $D_{\mathrm{ck}}$. The notation $\varepsilon_{2}$ should also be changed to $\varepsilon_{\text {intra }}$. Correspondingly, eqs. (D.50), (D.51) should be amended as follows:

\section{Modified equations in appendix D.2.4}

$$
\begin{aligned}
\mathcal{E}= & G_{1}\left(\widehat{\operatorname{dev}}\left(\varepsilon_{1}\right)\right)^{2}+G_{2}\left(\widehat{\operatorname{dev}}\left(\varepsilon_{\text {intra }}\right)\right)^{2} \\
\mathcal{D}= & \eta_{\text {cyto }}\left(\operatorname{dev} D_{\text {intra }}-\operatorname{dev} D_{1}\right)^{2}+\sigma_{\mathrm{Y}}\left|\operatorname{dev} D-\operatorname{dev} D_{\text {intra }}-\operatorname{dev} D_{3}-\operatorname{dev} D_{\text {ck }}\right| \\
& +\eta_{3}\left(\operatorname{dev} D_{3}\right)^{2}+\frac{1}{2} \eta_{\text {sw }}\left(\operatorname{tr} D_{\text {intra }}-r_{\text {sw }}+\operatorname{tr} D_{\text {ck }}\right)^{2} \\
& +\frac{1}{2} \eta_{\text {apo }}\left(\operatorname{tr} D-\operatorname{tr} D_{\text {intra }}-\operatorname{tr} D_{\text {ck }}+r_{\text {apo }}\right)^{2}
\end{aligned}
$$

In appendix D.2.4 entitled "Complex example", as a result of the restricted validity of eqs. (D.37), (D.39), the sentence after eq. (D.54) should be reformulated as follows:

\section{Modified sentence in appendix D.2.4}

Equations (14), (22), (D.29), (D.30), (D.50)-(D.54) close the set of equations which determine the evolution of the tissue. 